\title{
基于胆甾相液晶的可调制光子晶体
}

\author{
韩国志* $, a, b$ 朱沈 ${ }^{b}$ 吴生蓉 ${ }^{b}$ 庞峰飞 $b$ \\ ( ${ }^{a}$ 东南大学分子电子学国家重点实验室 南京 210096) \\ ( ${ }^{b}$ 南京工业大学理学院应用化学系 南京 210009)
}

\begin{abstract}
摘要 将胆甾相液晶填充进胶体晶体内部空隙, 通过胆甾相液晶与胶体晶体的耦合, 构建了一种新型可调制液晶光子 晶体. 填充于胶体晶体内部的胆甾相液晶织构呈现典型的手性近晶相(S)特征. 由于胆甾相液晶具有特定的选择性反 射, 当胶体晶体的带隙处于胆甾相液晶的反射波长范围之内, 则随着温度的改变, 胶体晶体的带隙与胆甾相液晶的带 隙同时发生蓝移. 在一定温度条件下, 胆甾相液晶的带隙将与胶体晶体的带隙发生塊合, 实现了光子晶体带隙在单峰 与双峰之间的可逆切换.
\end{abstract}

关键词 胆甾相液晶; 胶体晶体; 可调制; 光子晶体; 双带隙

\section{Preparation of Tunable Photonic Crystal Based on Cholesteric Liquid Crystal}

\author{
Han, Guozhi* ${ }^{*, a} \quad$ Zhu, Shen ${ }^{b} \quad$ Wu, Shengrong ${ }^{b} \quad$ Pang, Fengfei ${ }^{b}$ \\ ( ${ }^{a}$ State Key Laboratory of Bioelectronics, Southeast University, Nanjing 210096, China) \\ ( ${ }^{b}$ Department of Applied Chemistry, Nanjing University of Technology, Nanjing 210009, China)
}

\begin{abstract}
Via introducing cholesteric liquid crystal into voids of colloidal crystal, a novel tunable photonic colloidal crystal based on coupling of cholesteric liquid crystal (CLC) and colloidal crystal (CC) was prepared. Cholesteric liquid crystal used in this paper was prepared by combining nematic liquid crystal $5 \mathrm{CB}$ and the chiral dopant $\mathrm{COC}$ (cholesteryl oleyl carbonate), its selective reflection wavelength can be controlled by changing proportion of the mixture, and with temperature rising, blue shift of the selective reflection peak occurred. Firstly, $\mathrm{SiO}_{2}$ colloidal crystal was obtained by deposition method, then cholesteric liquid crystals was filled in voids of colloidal crystal by capillary force with temperature above the clearing point. Under polarized light microscope, the cholesteric liquid crystals in voids of colloidal crystal exhibited typical texture of chiral smectic phase in certain temperature region. Since cholesteric liquid crystal has particular selective reflection, if band gap of colloidal crystal locates in the reflection wavelength region of cholesteric liquid crystal, homodromous shift of band gap of cholesteric liquid crystal and colloidal crystal originating from changing of refraction index of cholesteric liquid crystal in voids occurred at the same time with temperature changed. But shift rate of band gap of cholesteric liquid crystal was faster than that of colloid crystal. So when temperature was carefully raised from lower limit of working range of cholesteric liquid crystal, band gap of CLC would gradually approach reflection peak of colloid crystal. Under a certain temperature, band gap of colloidal crystal would couple with selective reflection of CLC and form a single peak, reflection intensity increased from an average of $50 \%$ to $74 \%$. With the further increase of temperature, peak of selective reflection of CLC exceeded stop-band of colloid crystal, the single reflection peak re-splitted into double band. Then reversible transition between single and double stop-band of photonic crystal device was realized.
\end{abstract}

Keywords cholesteric liquid crystal; colloidal crystal; tunable; photonic crystal; double band gap

\section{1 引言}

光子晶体具有光子带隙的周期性电介质结构, 落在 光子带隙中的光将不能传播, 由此产生可抑制或增强自 发辐射的特性, 使之成为功能光学材料领域的热点 ${ }^{[1 \sim 4]}$. 光子晶体一个主要研究热点是可调制性能的实现, 即通 过外部条件的刺激，促使光子晶体带隙的可逆改变 ${ }^{[510]}$. 目前报道较多的光子晶体调制形式主要有两种, 一种是 光子带隙的移动, 另一种是光子带隙的开关. 在此领域
的研究中，由于液晶具有良好的对外场响应的特性，液 晶与光子晶体的耦合是实现可调制光子晶体的一个重 要手段. 将液晶填充到光子晶体中的空隙, 通过调控液 晶分子的取向或者相变，可以制备对外场响应的可调制 光子晶体. 在此类研究中, 采用的液晶主要是向列相小 分子液晶, 以及单个带隙的调制 ${ }^{[11}$ 17]. 而本文研究胆甾 相液晶与光子材料的耦合, 创新性地利用胆甾相液晶所 特有的选择性反射, 制备了可调制双带隙液晶光子晶体. 在一定的条件下, 其带隙能实现双峰-单峰的可逆转变,

* E-mail: han@njut.edu.cn

Received March 22, 2012; published August 17, 2012.

Supporting information for this article is available free of charge via the Internet at http://sioc-journal.cn.

Project supported by China Postdoctoral Foundation (No. 20100481084).

项目受中国博士后基金(No. 20100481084)资助. 
拓展了光子晶体的调制模式, 在波分复用, 光子传输、 激光等领域有着广阔的应用前景.

\section{2 结果与讨论}

\section{1 胆甾相液晶的光学特性}

由于本文中的胆甾相液晶是向列液晶 $5 \mathrm{CB}$ 和胆甾 醇油烯基碳酸酯(COC)的混合物, 其光学特性受两者比 例影响, 因此可方便地调节胆甾相液晶选择性反射光的 波长范围. 胆甾醇碳酸酯的结构如图 1 所示.

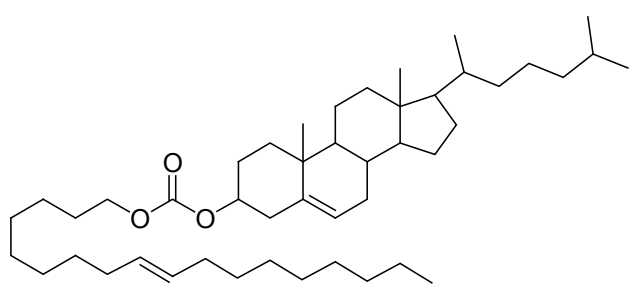

图 1 胆甾醇碳酸酯的结构

Figure 1 Molecular structure of cholesteryl oleyl carbonate

实验测得其工作区间为 $20 \sim 36{ }^{\circ} \mathrm{C}$, 对于含有 $30 \%$ $5 \mathrm{CB}$ 的胆甾相液晶而言, 其禁带范围为 $550 \sim 720 \mathrm{~nm}$. 对于含有 $50 \% 5 \mathrm{CB}$ 的胆甾相液晶而言, 其禁带范围为 $580 \sim 740 \mathrm{~nm}$. 当 $5 \mathrm{CB}$ 的含量增大到 70\%, 其禁带消失. 此外, 胆甾相液晶的布拉格反射通常随着温度的升高而 蓝移. 图 2 为两种 $5 \mathrm{CB}$ 浓度的胆甾相液晶的反射光谱.

实验结果显示, 胆甾相液晶在禁带部分的透光率随 着禁带的蓝移而升高. 因此, 在蓝移的过程中, 其反射 率通常略有下降. 同时, 实验测得, 胆甾相液晶的平均 折射率随着温度的升高而逐渐减小，如表 1 所示.

表 1 胆甾相液晶平均折射率随温度的变化

Table 1 Average refractive index of cholesteric liquid crystal at different temperatures

\begin{tabular}{ccccc}
\hline Isotropic phase & $32{ }^{\circ} \mathrm{C}$ & $28{ }^{\circ} \mathrm{C}$ & $24{ }^{\circ} \mathrm{C}$ & Solid phase \\
\hline 1.518 & 1.5220 & 1.522 & 1.526 & 1.600 \\
\hline
\end{tabular}

将胆甾相液晶填充进胶体晶体空隙中之后, 采用偏 光显微镜分析处于 $\mathrm{SiO}_{2}$ 胶体晶体内部胆甾相液晶的微 观结构. 图 3 是单纯的胆甾相液晶与填充于胶体晶体内 部的胆甾相液晶的织构对比(液晶基片平行取向).

与单纯的胆甾相液晶相比, 填充于胶体晶体内部的 胆甾相液晶的织构呈现典型的碎扇型. 属于手性近晶相 (S)的特征. 表明胶体晶体的周期性结构对其内部胆甾 相液晶的分子排列有着很大的影响，在其诱导下，胆甾 相液晶依然能形成周期性排列.

\section{2 可调制光子晶体的光学性能}

胶体晶体的带隙可以按照下列公式进行计算:

$$
\lambda=2(2 / 3)^{1 / 2} d\left(n_{\mathrm{a}}^{2}-\sin ^{2} \theta\right)^{1 / 2}
$$

$$
n_{\mathrm{a}}^{2}=n_{\text {void }}^{2} f_{\text {void }}+n_{\text {sphere }}^{2} f_{\text {sphere }}
$$

其中 $d$ 代表两个最近邻粒子间的中心距离, $\theta$ 为光波的 入射角度, $n_{\mathrm{a}}$ 为体系的平均折射率, $n_{\mathrm{void}}$ 和 $n_{\text {sphere }}$ 分别是 空隙材料和粒子的折射率, $f_{\text {void }}$ 和 $f_{\text {sphere }}$ 是空隙和粒子在 光子晶体中占有的体积比.
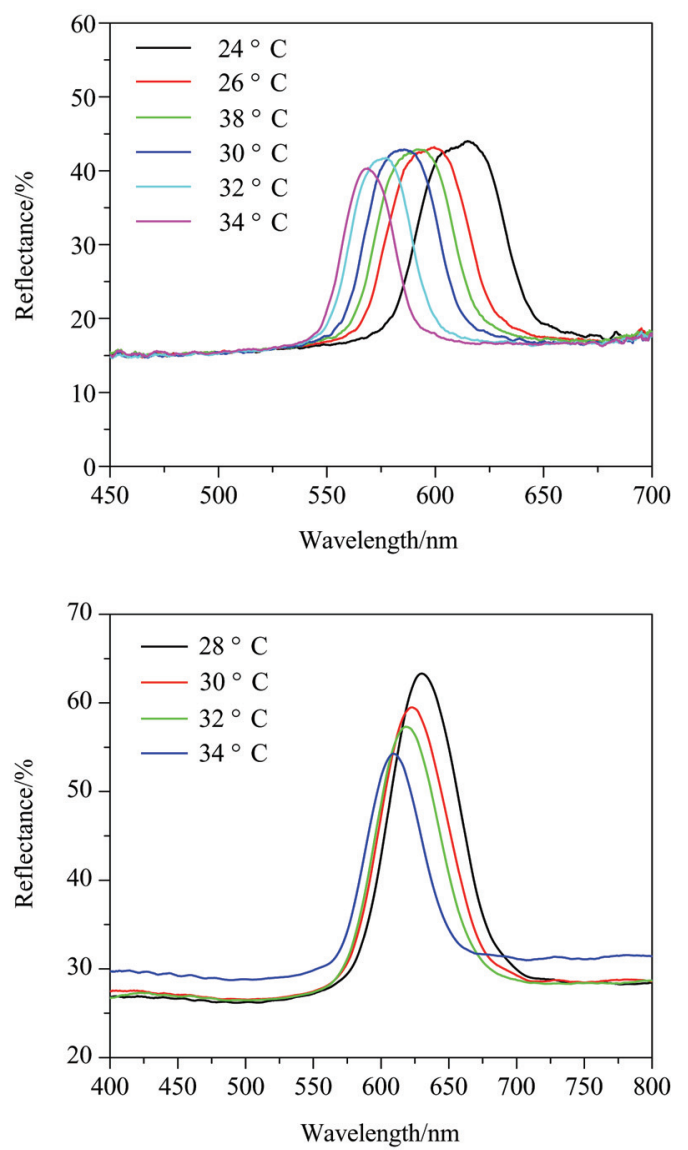

图 2 不同 $5 \mathrm{CB}$ 比例的胆甾相液晶的反射光谱

Figure 2 Reflection spectra of cholesteric liquid crystals with different proportion of $5 \mathrm{CB}$

(a) $30 \%$; (b) $50 \%$

当胶体晶体填充胆甾相液晶以后，其反射峰发生 了大约 $70 \mathrm{~nm}$ 左右的红移. 图 4 显示的是直径为 $240 \mathrm{~nm}$ $\mathrm{SiO}_{2}$ 胶体晶体填充胆甾相液晶前后反射峰的对比. 其中 液晶处于低温固态. 没有布拉格反射.

将光子晶体置于热台上，随着温度的升高，胆甾相 液晶由固态进入液晶态, 光子晶体逐渐呈现两个反射峰, 如图 5 所示.

随着温度进一步升高，胆甾相液晶的反射波长逐 渐蓝移, 其蓝移幅度为 $4 \mathrm{~nm} /{ }^{\circ} \mathrm{C}$, 在此过程中, 胶体晶体 所呈现的布拉格反射也同方向逐渐移动，因为在此过程 中, 胆甾相液晶的平均折射率逐渐减小, 但变化不大, 导致胶体晶体本身的带隙移动速度及幅度较小, 因此, 随着温度的升高, 在某一时刻, 胆甾相液晶的反射峰将 

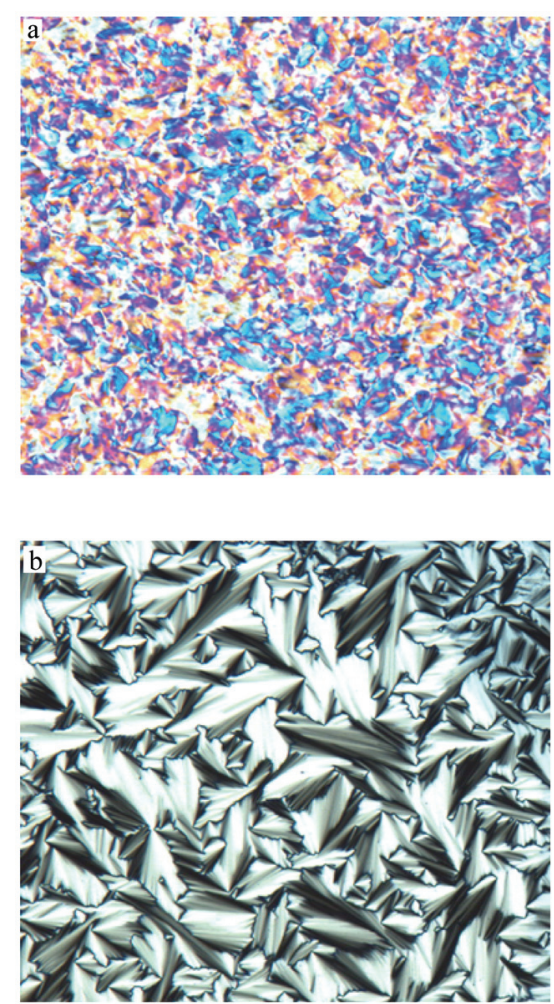

图 3 单纯的胆甾相液晶的织构(a)与填充于胶体晶体内部时的织构 (b)

Figure 3 Texture of pure cholesteric liquid crystals (a) and in voids of $\mathrm{SiO}_{2}$ colloidal crystal (b)

$22{ }^{\circ} \mathrm{C}, 5 \mathrm{CB}: 30 \%$

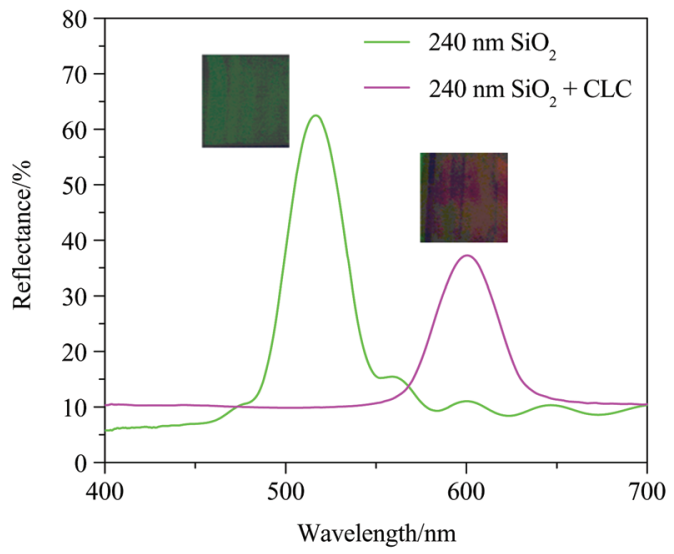

图 $4 \mathrm{SiO}_{2}$ 胶体晶体填充胆甾相液晶前后的反射光谱 $\left(16{ }^{\circ} \mathrm{C}\right)$

Figure 4 Shift of reflection peak of $\mathrm{SiO}_{2}$ colloidal crystal doped with CLC $\left(16{ }^{\circ} \mathrm{C}\right)$

追上胶体晶体的反射峰并发生耦合. 反射强度大大增强, 从平均 50\%增大到 74\%左右. 如图 6 所示, 当温度进一 步升高, 胆甾相液晶禁带较快的蓝移使得其超越胶体晶 体的禁带, 重叠增强的单峰再次发生分裂, 形成各自的 单峰. 分裂之后, 胶体晶体禁带的反射强度有所降低, 那是由于胶体晶体微球与胆甾相液晶的折射率差缩小 的原因. 当温度超过胆甾相液晶的清亮点, 则胆甾相液 晶的反射峰消失, 双峰再次变成单峰.

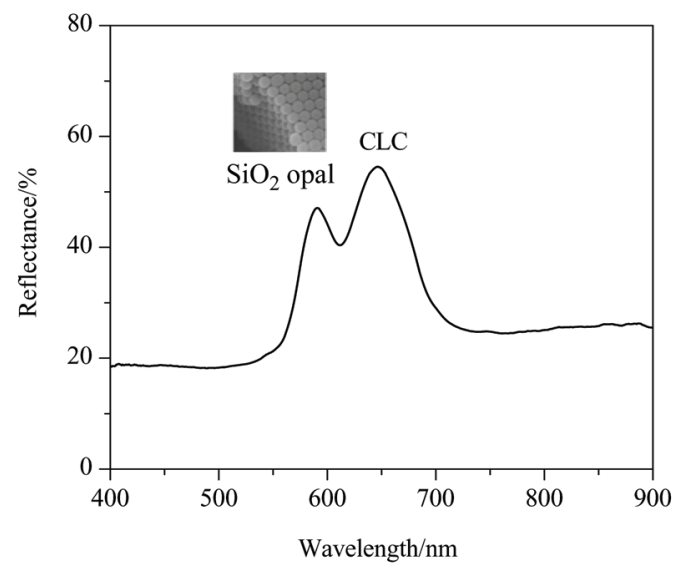

图 5 填充胆甾相液晶的 $\mathrm{SiO}_{2}$ 胶体晶体的反射光谱 $\left(22{ }^{\circ} \mathrm{C}\right)$

Figure 5 Reflection spectra of $\mathrm{SiO}_{2}$ colloidal crystal doped with CLC $\left(22{ }^{\circ} \mathrm{C}\right)$

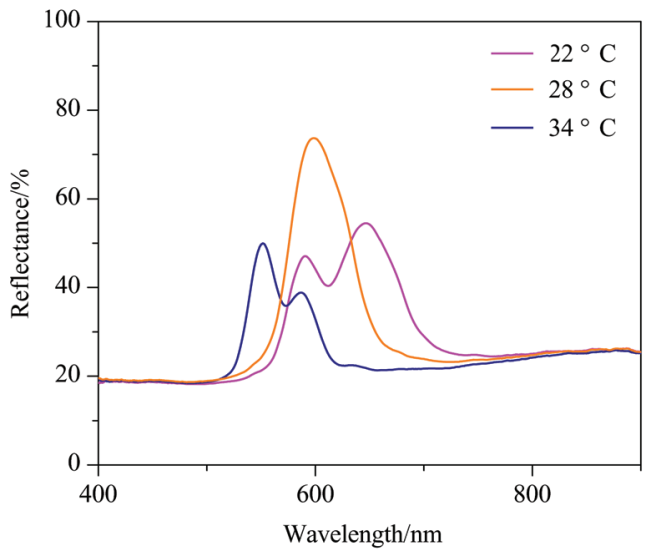

图 6 双峰光子晶体反射光谱随温度的变化

Figure 6 Reflection spectra of double-band photonic crystal at various temperatures

因此, 随着温度的改变, 光子晶体的反射峰形成了 双峰一单峰-双峰的循环可逆转变. 对于制备波分复用光 子器件以及激光器件提供了很好的思路，由于耦合单峰 的波长主要依赖于胶体晶体本身的选择性反射，而胶体 晶体的选择性反射与晶面间距和材料的折射率相关，因 此，此光子晶体的光学特性可以通过调节胶体晶体微球 的材料或直径来调节.

\section{3 结论}

采用向列液晶 $5 \mathrm{CB}$ 和手性物质胆甾醇油烯基碳酸 酯制备了胆甾相液晶. 将胆甾相液晶填充进 $\mathrm{SiO}_{2}$ 胶体 晶体内部空隙, 通过胆甾相液晶选择性反射和 $\mathrm{SiO}_{2}$ 胶 体晶体光子带隙的耦合, 制备了双带隙光子晶体. 在一 定的温度范围内, 可以实现双峰-单峰-双峰的可逆调制. 在波分复用、光子传输等领域有着巨大的应用前景.

\section{4 实验部分}

\section{1 胆甾相液晶的制备}

将胆甾醇碳酸酯加热熔化，然后加入一定量的向列 
液晶 $5 \mathrm{CB}$, 然后超声分散 $10 \mathrm{~min}$, 冷却至室温, 形成白 色粘稠状液体. 然后取少量夹在两片玻片之间, 置于程 序控制热台之上, 从室温开始逐渐升温, 用光纤光谱仪 实时检测其反射光谱. 用阿贝折光仪测量不同温度下胆 甾相液晶的平均折射率.

\section{2 可调制晶体的制备}

利用沉积法制备 $\mathrm{SiO}_{2}$ 胶体晶体, 用扫描电镜分析 其形貌. 然后将胆甾相液晶加热至透明状液体, 利用毛 细管作用力将胆甾相液晶填充进 $\mathrm{SiO}_{2}$ 胶体晶体内部空 隙. 用透明石英片封盖. 冷却至室温. 然后程序升温, 在此过程中, 用光纤光谱仪实时监测其反射光谱的变化. 同时，采用偏光显微镜分析其微观织构.

\section{References}

[1] Joannopoulos, J. D.; Meade, R. D.; Winn, J. N. Photonic Crystals, Princeton University Press, Princeton, NJ, 1995.

[2] Zhao, X.-W.; Cao, Y.; Ito, F.; Chen, H.-H.; Nagai, K.; Zhao, Y.-Y.; Gu, Z.-Z. Angew. Chem. Int. Ed. 2006, 45, 6835.
[3] Cassagneau, T.; Caruso, F. Adv. Mater. 2002, 14, 1629

[4] Ge, H.; Wang, G.; He, Y.; Wang, X.; Song, Y.; Jiang, L.; Zhu, D. ChemPhysChem 2006, 7, 575

[5] Han, G.-Z.; Xie, Z.-Y.; Zheng, D.; Sun, L.-G.; Gu, Z.-Z. Appl. Phys. Lett. 2007, 91, 141114.

[6] Xie, Z.-Y.; Sun, L.-G.; Han, G.-Z.; Gu, Z.-Z. Adv. Mater. 2008, 20 , 3601 .

[7] Kubo, S.; Gu, Z.-Z.; Takahashi, K.; Fujishima, A.; Segawa, H.; Sato, O. J. Am. Chem. Soc. 2004, 126, 8314

[8] McPhail, D.; Straub, M.; Gu, M. Appl. Phys. Lett. 2005, 86, 051103.

[9] Gu, Z.-Z.; Iyoda, T.; Fujishima, A.; Sato, O. Adv. Mater. 2001, 13, 1295.

[10] Han, G.-Z.; Xiong, G.-R.; Xie, Z.-Y.; Sun, L.-G. Chemistry 2010, 73, 332. (韩国志, 熊桂荣, 谢卓颖, 孙立国, 化学通报, 2010, 73, 332.)

[11] Ozaki, M.; Matsuhisa, Y.; Yoshida, H.; Ozaki, R.; Fujishima, A. Phys. Status Solidi A 2007, 204, 3777.

[12] Payne, J. C.; Thomas, E. L. Adv. Funct. Mater. 2007, 17, 2717.

[13] Shirota, K.; Sun, H.; Kawata, S. Appl. Phys. Lett. 2004, 84, 1632.

[14] Jiang, P.; Bertone, J. F.; Hang, K. S.; Colvin, V. L. Chem. Mater. 1999, 11, 2132.

[15] Lopez, C. Adv. Mater. 2003, 15, 1679.

[16] Qian, W.-P.; Gu, Z.-Z.; Fujishima, A.; Sato, O. Langmuir 2002, 18, 4526.

[17] Gu, Z.-Z.; Kubo, S.; Qian, W.-P.; Einaga, Y.; Tryk, D. A.; Fujishima, A.; Sato, O. Langmuir 2001, 17, 6751.

[18] Stober, W.; Fink, A.; Bohn, E. J. Colloid Interface Sci. 1968, 26, 62.

(Cheng, B.; Fan, Y.) 